\title{
Using Factorial Experimental Design to Prepare Size-Tuned ${ }_{2}$ Nanovesicles
}

\author{
3 Pablo García-Manrique, ${ }^{\dagger, \ddagger}$ María Matos, ${ }^{\dagger}$ Gemma Gutiérrez, ${ }^{\dagger}$ Oscar R. Estupiñán, ${ }^{\dagger, \ddagger}$ \\ ${ }_{4}$ María Carmen Blanco-Lopez, ${ }^{\ddagger}$ and Carmen Pazos*, ${ }^{*}$ \\ $5{ }^{\dagger}$ Department of Chemical and Environmental Engineering and ${ }^{\star}$ Department of Physical and Analytical Chemistry, University of \\ 6 Oviedo, Julián Clavería 8, 33006 Oviedo, Spain
}

\section{S Supporting Information}

8 ABSTRACT: The aim of this work was to prepare size-tuned 9 nanovesicles using a modified ethanol injection method (EIM) 10 by applying factorial experimental design. Stable size-tuned 11 nanovesicles (liposomes and niosomes) with controlled sizes 12 and high EE values for hydrophobic compounds (Sudan Red $137 \mathrm{~B}$ and vitamin $\mathrm{D}_{3}$ ) were achieved. Equations that were able to 14 predict the mean particle sizes, in the ranges of 55-156 nm for 15 liposomes and 224-362 $\mathrm{nm}$ for niosomes with PDI values 16 between 0.032 and 0.378 , were obtained. These customized 17 soft nanoparticles could be suitable in food, cosmetic, pharmace

\section{INTRODUCTION}

18 Controlled preparation of nanoparticles has attracted great 19 interest in recent years. ${ }^{1}$ Nanovesicles are an important family of 20 organic nanoparticles, produced by bottom-up nanotechnology, 21 with relevant applications in biomedicine, ${ }^{2}$ food science, ${ }^{3}$ 22 analytical chemistry, ${ }^{4,5}$ and biosensors. ${ }^{6}$ They are considered 23 soft nanoparticles because interactions among their molecular 24 components are similar to those arising from biological systems. ${ }^{7}$ 25 Most of the work describing their preparation for specific uses 26 has focused on the optimization of their composition with the 27 aim of maximizing encapsulation efficiency, delivery, or delivery 28 control.

29 However, size is one of the most critical properties (together 30 with shape and surface chemistry) for understanding cell-uptake 31 processes and, therefore, bioavailability and targetability. ${ }^{7}$ Several 32 studies have focused on the optimization of the drug 33 encapsulation efficiency while considering size as just a property 34 for controlling administration parameters, such as penetration 35 kinetics in topical formulations. For example, Padamwar et al. ${ }^{8}$ 36 studied the encapsulation of vitamin $\mathrm{E}$ in liposomes and found 37 that the amount of lipids yielded a positive correlation with size, 38 which was, in turn, negatively correlated with penetration 39 efficiency into the skin. Sometimes, size has been found to 40 increase with higher amounts of membrane components, such as 41 cholesterol, whereas it decreased with higher amounts of 42 surfactants (e.g., Tween 80). Simultaneously, cholesterol or 43 surfactants can affect encapsulation efficiency (EE). Optimal 44 situations can be reached as a compromise at intermediate levels 45 of both factors. In that case, Taha ${ }^{9}$ also reported an interaction 46 between membrane-component concentration and size reduc47 tion by ultrasound, making factor optimization an essential task. 48 In other cases, an opposite effect was observed, and higher 49 concentrations of membrane components (such as Span 60 and cholesterol) produced larger sizes and increased EEs. It is useful 50 to deliver efficient amounts of a selected drug into superficial skin 51 layers without systemic absorption. ${ }^{10}$ On this basis, the goal of 52 our work was to set up a bulk method for producing nanovesicles 53 of controlled size that could be subsequently modified for specific 54 applications.

Vesicles are colloidal particles in which a concentric bilayer 56 made up of amphiphilic molecules surrounds an aqueous 57 compartment. These vesicles are commonly used to encapsulate 58 both hydrophilic and lipophilic compounds, for food, cosmetic, 59 pharmaceutical, or medical applications, such as diagnosis or 60 therapy. ${ }^{11}$ Hydrophilic compounds are entrapped into the 61 aqueous compartments between bilayers, whereas lipophilic 62 compounds are preferentially located inside the bilayers. ${ }^{12,13}$ The 63 most common types of vesicles are liposomes and niosomes. 64

Liposomes were first described by Bangham et al. in 1965, ${ }^{14} 65$ and they are basically spherical bilayer vesicles formed by the self- 66 assembly of phospholipids. This self-assembly process is based 67 on the interactions occurring between phospholipids and water 68 molecules, where the polar head groups of phospholipids are 69 exposed to the aqueous phases (inner and outer) and the 70 hydrophobic hydrocarbon tails are forced to face each other in a 71 bilayer. ${ }^{15}$ Because of the presence of both lipid and aqueous 72 phases in liposome structures, they can be used for the 73 encapsulation, delivery, and controlled release of hydrophilic, 74 lipophilic, and amphiphilic compounds. ${ }^{15,16} 75$

On the other hand, niosomes are vesicles formed by the self- 76 assembly of nonionic surfactants in aqueous media resulting in 77

Received: April 21, 2016

Revised: July 28, 2016

Accepted: August 10, 2016 
Table 1. Plackett-Burman Fractional Factorial Design: Responses, Levels, and Factors

\begin{tabular}{|c|c|c|c|c|c|c|c|c|c|c|c|c|c|}
\hline \multicolumn{7}{|c|}{ response code } & \multicolumn{7}{|c|}{ meaning } \\
\hline & \multicolumn{5}{|c|}{$Y_{1}$} & & \multicolumn{7}{|c|}{$\begin{array}{l}\text { Z-average size of PC liposomes } \\
\text { PDI of PC liposomes }\end{array}$} \\
\hline \multirow[b]{3}{*}{ level } & \multicolumn{13}{|c|}{ factors } \\
\hline & \multicolumn{4}{|c|}{ formulation } & \multicolumn{4}{|c|}{ injection } & \multicolumn{3}{|c|}{ evaporation } & \multicolumn{2}{|c|}{ sonication } \\
\hline & $\mathrm{O} / \mathrm{A}\left(X_{1}\right)$ & & $\left(X_{2}\right)(g / L)$ & $I\left(X_{3}\right)(\mathrm{mM})$ & $\mathrm{Q}_{\mathrm{V}}\left(X_{4}\right)(\mathrm{mL} / \mathrm{h})$ & ) $T$ & ${ }_{\mathrm{I}}\left(X_{5}\right)\left({ }^{\circ} \mathrm{C}\right)$ & $N_{\mathrm{S}}\left(X_{6}\right)(\mathrm{rpm})$ & $T_{\mathrm{E}}\left(X_{7}\right)\left({ }^{\circ} \mathrm{C}\right)$ & $N_{\mathrm{R}}$ & $\mathrm{pm})$ & $A\left(X_{9}\right)(\%)$ & $t\left(X_{10}\right)(\mathrm{min})$ \\
\hline-1 & $5: 50$ & & 2.5 & 10 & 50 & & 30 & 350 & 35 & & & 25 & 15 \\
\hline 1 & $20: 50$ & & 6.0 & 150 & 215 & & 60 & 900 & 60 & & & 42 & 30 \\
\hline batcl & & $X_{1}$ & $X_{2}$ & $X_{3}$ & $X_{4}$ & $X_{5}$ & $X_{6}$ & $X_{7}$ & $X_{8}$ & $X_{9}$ & $X_{10}$ & $Y_{1}$ & $Y_{2}$ \\
\hline PB1 & & 1 & 1 & -1 & -1 & -1 & -1 & 1 & -1 & 1 & 1 & 90 & 0.254 \\
\hline PB2 & & 1 & -1 & -1 & 1 & -1 & 1 & 1 & 1 & 1 & -1 & 93 & 0.129 \\
\hline PB3 & & -1 & -1 & -1 & -1 & -1 & -1 & 1 & 1 & -1 & -1 & 97 & 0.152 \\
\hline PB4 & & 1 & -1 & 1 & -1 & 1 & 0 & 1 & -1 & 1 & -1 & 72 & 0.205 \\
\hline PB5 & & -1 & 1 & -1 & 1 & -1 & -1 & 1 & -1 & -1 & 1 & 258 & 0.413 \\
\hline PB6 & & 1 & 1 & 1 & 1 & -1 & -1 & -1 & 1 & -1 & -1 & 102 & 0.176 \\
\hline PB7 & & -1 & -1 & 1 & 1 & -1 & -1 & -1 & -1 & 1 & 1 & 84 & 0.218 \\
\hline PB8 & & 1 & -1 & -1 & 1 & 1 & 1 & -1 & 1 & -1 & 1 & 106 & 0.240 \\
\hline PB9 & & -1 & -1 & -1 & -1 & 1 & 1 & -1 & 1 & 1 & 1 & 71 & 0.229 \\
\hline PB1C & & 1 & -1 & 1 & -1 & -1 & -1 & -1 & -1 & -1 & 1 & 81 & 0.141 \\
\hline PB1 & & -1 & 1 & 1 & -1 & 1 & -1 & 1 & 1 & -1 & 1 & 152 & 0.316 \\
\hline $\mathrm{PB} 12$ & & 1 & 1 & 1 & 1 & 1 & 1 & 1 & 1 & 1 & 1 & 65 & 0.260 \\
\hline $\mathrm{PB} 1$ & & -1 & -1 & 1 & 1 & 1 & 1 & 1 & -1 & -1 & -1 & 87 & 0.189 \\
\hline $\mathrm{PB} 1$ & & -1 & 1 & 1 & -1 & -1 & 1 & -1 & 1 & 1 & -1 & 115 & 0.273 \\
\hline PB1 & & 1 & 1 & -1 & -1 & 1 & 1 & -1 & -1 & -1 & -1 & 113 & 0.199 \\
\hline PB1C & & -1 & 1 & -1 & 1 & 1 & -1 & -1 & -1 & 1 & -1 & 74 & 0.271 \\
\hline
\end{tabular}

78 closed bilayer structures. ${ }^{13,17,18}$ As liposomes, their formation 79 process is a consequence of unfavorable interactions between 80 surfactants and water molecules, and they can also entrap 81 hydrophilic, lipophilic, and amphiphilic compounds. ${ }^{19,20}$

82 Niosomes exhibit a number of advantages over liposomes, 83 such as higher stability, easy access to raw materials, lower 84 toxicity, high compatibility with biological systems, non85 immunogenicity, and versatility for surface modification. ${ }^{20}$

86 Cholesterol is commonly used as a membrane additive for 87 nanovesicle preparation to improve vesicle stability, entrapment 88 efficiency, and release under storage. ${ }^{20}$ It increases vesicle size 89 and rigidity, improving encapsulation efficiency, but at high 90 concentrations, it can adversely affect the encapsulation rate. ${ }^{21,22}$ 91 Cholesterol also plays a fundamental role in niosome formation 92 when hydrophilic surfactants are used (hydrophile/lipophile 93 balance of $\sim 10)$. $^{20}$

94 More than 20 different methods have been identified for 95 nanovesicle preparation, and these methods were recently 96 reviewed. ${ }^{23,24}$ In this work, a modified ethanol injection method 97 (EIM) is used, because it offers some advantages over other 98 methods, such as simplicity, absence of potentially harmful 99 chemicals, and suitability for scaleup. ${ }^{25}$

100 The conventional EIM was first described in $1973 .^{26}$ In this 101 technique, lipids/surfactants and additives are first dissolved in 102 an organic solvent, such as diethyl ether or ethanol, and then 103 injected slowly through a syringe into an aqueous phase 104 containing the compound of interest. Then, the organic solvent 105 is removed using a vacuum rotary evaporator. When ethanol is 106 used as the organic solvent, the spontaneous formation of 107 vesicles occurs as soon as the organic solution is in contact with 108 the aqueous phase, ${ }^{27}$ but vigorous agitation is needed to obtain 109 narrow size distributions. For this purpose, a final sonication 110 stage was applied in this study after organic-phase removal by 111 vacuum evaporation.
However, a large number of variables are involved in this 112 modified EIM, and selection of the most important of them 113 (screening) is a crucial step in rationally preparing vesicles by this 114 versatile method. In this work, the $Z$-average size and 115 polydispersity index (PDI) were selected as the dependent 116 variables. They are considered to be of great importance in 117 nanovesicle design because most of the final applications of these 118 vesicular systems are directly related to these two parameters. 119 Factorial experimental design and the analysis of variance 120 (ANOVA) methodology are appropriate and efficient statistical 121 tools that permit the effects of several factors that influence 122 responses to be studied by varying the factors simultaneously in a 123 limited number of experiments.

In the recent past, design of experiments (DoE) has been 125 extensively used for the study and optimization of vesicles and 126 other similar organic materials. Different designs can be applied 127 to reduce the number of factors involved in the preparation 128 techniques $^{28}$ and, therefore, to minimize the number of 129 experiments without losing valuable information. Plackett- 130 Burman design is a type of fractional design involving relatively 131 few runs, ${ }^{29}$ commonly used for the screening of variables. 132

Another important role of $\mathrm{DoE}$ is in the optimization of 133 nanovesicle composition for the enhancement of intended 134 purposes. For instance, it has been applied to the formulation of 135 liposomes (phospholipid and cholesterol ratio) for the topical 136 delivery of vitamin $\mathrm{E}^{8}$ hybrid liposomes (with both low- and 137 high-transition-temperature phospholipids) to improve the 138 encapsulation and delivery of silymarin, ${ }^{30}$ and niosomes for 139 topical delivery applications. ${ }^{10,31} \mathrm{DoE}$ has also been used to 140 enhance the transdermal flux of raloxifene hydrochloride ${ }^{32}$ and 141 diclofenac diethylamine ${ }^{33}$ loaded transfersomes and of other 142 polymeric nanoparticles encapsulating an anticancer drug. ${ }^{34} 143$ Moreover, the interactions between vesicles and proteins, such as 144 pectin, to improve drug-delivery properties has been studied by 145 
146 DoE. $^{35}$ Nanostructured lipid carriers (NLCs) loaded with 147 flurbiprofen were also produced under optimal conditions 148 using full factorial design. ${ }^{36}$

149 In this work, an initial fractional factorial design with two levels 150 (Plackett-Burman) was used to screen the most important 151 factors in vesicle preparation by the EIM. Then, a $2^{3}$ two-level full 152 factorial design using center-point replicates was applied to study 153 the influence of the main factors and their interactions on the $Z$ 154 average size and PDI. Once the appropriate operating conditions 155 were determined, vesicle stability was studied by using multiple 156 light scattering technology and by measuring the encapsulation 157 efficiencies (EEs) of different compounds.

\section{MATERIALS AND METHODS}

158 2.1. Materials. Phosphatidylcholine (PC) (predominant 159 species $\mathrm{C}_{42} \mathrm{H}_{80} \mathrm{NO}_{8} \mathrm{P}, \mathrm{MW}=775.04 \mathrm{~g} / \mathrm{mol}$ ) from soybean 160 (Phospholipon 90G) was a kind gift from Lipoid (Ludwigshafen, 161 Germany). Sorbitan monostearate (Span 60, S60) $\left(\mathrm{C}_{24} \mathrm{H}_{46} \mathrm{O}_{6}\right.$, $162 \mathrm{MW}=430.62 \mathrm{~g} / \mathrm{mol})$ and cholesterol $(\mathrm{Cho})\left(\mathrm{C}_{27} \mathrm{H}_{46} \mathrm{O}, \mathrm{MW}=\right.$ $163386.65 \mathrm{~g} / \mathrm{mol}$ ) were purchased from Sigma-Aldrich (St. Louis, $164 \mathrm{MO})$. All membrane components were dissolved in absolute 165 ethanol (Sigma-Aldrich, St. Louis, MO).

166 Methanol, acetonitrile, 2-propanol, and acetic acid of high167 performance liquid chromatography (HPLC) grade were 168 supplied by Sigma-Aldrich (St. Louis, MO).

169 A phosphate buffer (PB) solution (10 mM, pH 7.4) was used 170 in all experiments as the aqueous phase. The buffer solution was 171 prepared in Milli-Q water by dissolving proper amounts of 172 sodium dihydrogen phosphate and sodium hydrogen phosphate, 173 supplied by Panreac (Barcelona, Spain). Sodium chloride from 174 Panreac (Barcelona, Spain) was added to increase the ionic 175 strength when required according to the experiments listed in 176 Table 1. For the encapsulation experiments, Fat Red Bluish or 177 Sudan Red $7 \mathrm{~B}$ dye $\left(\mathrm{C}_{24} \mathrm{H}_{21} \mathrm{~N}_{5}, \mathrm{MW}=379.46 \mathrm{~g} / \mathrm{mol}\right)$ and 178 cholecalciferol or vitamin $\mathrm{D}_{3}\left(\mathrm{C}_{27} \mathrm{H}_{44} \mathrm{O}, \mathrm{MW}=384.64 \mathrm{~g} / \mathrm{mol}\right)$ 179 were purchased from Sigma-Aldrich (St. Louis, MO).

180 2.2. Factorial Design of Experiments. Factors that could 181 potentially affect the size of vesicles produced by the EIM were 182 classified in four groups, according to the different steps involved 183 in this preparation method: formulation (organic/aqueous phase 184 volume ratio, phospholipid concentration, and ionic strength), 185 injection (injection flow rate, temperature, and stirring speed), 186 evaporation (temperature and rotation speed), and sonication 187 (amplitude and time of sonication).

188 To identify the relative effects of variables on the response, a 189 two-level fractional factorial design was used. A Plackett190 Burman $(\mathrm{P}-\mathrm{B})$ resolution III design with $n=16$ runs was 191 proposed for screening of the initial factors. Two levels were 192 selected for each variable.

193 Table 1 lists the factors and levels involved in the $\mathrm{P}-\mathrm{B}$ 194 fractional factorial design used, where $\mathrm{O} / \mathrm{A}$ is the organic/ 195 aqueous phase volume ratio, $C$ is the concentration of 196 phospholipid, $I$ is the ionic strength, $Q_{V}$ is the injection flow 197 rate, $T_{\mathrm{I}}$ is the injection temperature, $N_{S}$ is the stirring speed 198 during injection, $T_{\mathrm{E}}$ is the evaporation temperature, $N_{\mathrm{E}}$ is the 199 evaporator rotation speed, $A$ is the sonication amplitude, and $t$ is 200 the sonication time.

201 In a second step, a $2^{3}$ full factorial design with center-point 202 repetitions $(n=5)$ was carried out to study the main effects and 203 interactions between factors previously selected by the screening 204 design (Table 2). All other factors were fixed at certain values. 205 In both designs, mean diameter ( $Z$-average size) and PDI were 206 selected as response variables. Minitab statistical software
Table 2. Full Factorial Design $\left(2^{3}\right)$ with Center-Point Repetitions $(n=5)$ : Factors, Levels, and Responses

\begin{tabular}{|c|c|c|c|}
\hline response code & \multicolumn{3}{|c|}{ meaning } \\
\hline$Y_{1}$ & \multicolumn{3}{|c|}{$Z$-average size of PC liposomes } \\
\hline$Y_{2}$ & \multicolumn{3}{|c|}{ PDI of PC liposomes } \\
\hline$Y_{3}$ & \multicolumn{3}{|c|}{$Z$-average size of $S 60:$ Cho niosomes } \\
\hline$Y_{4}$ & \multicolumn{3}{|c|}{ PDI of S60:Cho niosomes } \\
\hline & \multicolumn{3}{|c|}{ factors } \\
\hline level & $\mathrm{O} / \mathrm{A}\left(X_{1}\right)$ & $C\left(X_{2}\right)(\mathrm{g} / \mathrm{L})$ & $A\left(X_{3}\right)(\%)$ \\
\hline-1 (low) & $5: 50$ & 2 & 30 \\
\hline 0 (medium) & $12.5: 50$ & 5 & 42.5 \\
\hline 1 (high) & $20: 50$ & 8 & 55 \\
\hline
\end{tabular}

\begin{tabular}{lrrrrccc} 
batch & $X_{1}$ & $X_{2}$ & $X_{3}$ & $Y_{1}$ & $Y_{2}$ & $Y_{3}$ & $Y_{4}$ \\
FF1 & 1 & -1 & 1 & 65 & 0.299 & 305 & 0.075 \\
FF2 & 1 & 1 & -1 & 97 & 0.249 & 362 & 0.136 \\
FF3 & -1 & 1 & -1 & 149 & 0.296 & 294 & 0.206 \\
FF4 & -1 & 1 & 1 & 88 & 0.307 & 262 & 0.291 \\
FF5 & -1 & -1 & 1 & 64 & 0.342 & 242 & 0.120 \\
FF6 & 1 & 1 & -1 & 100 & 0.257 & 360 & 0.143 \\
FF7 & 1 & 1 & 1 & 64 & 0.272 & 241 & 0.182 \\
FF8 & -1 & -1 & -1 & 90 & 0.196 & 235 & 0.078 \\
FF9 & 0 & 0 & 0 & 82 & 0.219 & 301 & 0.195 \\
FF10 & 1 & -1 & -1 & 84 & 0.205 & 253 & 0.032 \\
FF11 & -1 & 1 & 1 & 107 & 0.297 & 276 & 0.235 \\
FF12 & -1 & 1 & -1 & 156 & 0.308 & 275 & 0.145 \\
FF13 & 1 & -1 & 1 & 65 & 0.378 & 248 & 0.066 \\
FF14 & 1 & -1 & -1 & 97 & 0.246 & 268 & 0.045 \\
FF15 & -1 & -1 & -1 & 84 & 0.173 & 239 & 0.094 \\
FF16 & 0 & 0 & 0 & 75 & 0.224 & 305 & 0.253 \\
FF17 & 0 & 0 & 0 & 84 & 0.250 & 317 & 0.118 \\
FF18 & 1 & 1 & 1 & 55 & 0.307 & 224 & 0.203 \\
FF19 & 0 & 0 & -1 & 77 & 0.242 & 308 & 0.241 \\
FF20 & 0 & 0 & 0 & 84 & 0.251 & 337 & 0.171 \\
FF21 & -1 & -1 & 1 & 69 & 0.343 & 233 & 0.124 \\
\hline & & & & & & &
\end{tabular}

(version 17) was used for all data analysis. Analysis of variance 207 (ANOVA) was used for this purpose.

208

Once the models were obtained taking into account significant 209 factors and interactions, a set of selected size-tuned vesicles were 210 prepared and characterized.

211

2.3. Vesicle Preparation. For liposome preparation, 212 appropriate weighed amounts of $\mathrm{PC}$ were dissolved in different 213 volumes of absolute ethanol (5-20 mL range). The same 214 procedure was applied to niosome preparation by weighing and 215 dissolving $\mathrm{S} 60$ and Cho in a 1:0.5 weight ratio. Then, the organic 216 solution was injected, with a syringe pump (KD Scientific, 217 Holliston, MA) at a flow rate of $120 \mathrm{~mL} / \mathrm{h}$, into Milli-Q water 218 that was kept at $60{ }^{\circ} \mathrm{C}$ and stirred at $500 \mathrm{rpm}$. Once vesicles 219 formed, ethanol was removed at $40{ }^{\circ} \mathrm{C}$ under reduced pressure 220 $(90 \mathrm{kPa})$ in a rotary evaporator. The resulting vesicular systems 221 were further sonicated for $15 \mathrm{~min}$ (CY-500 sonicator, Optic 222 Ivymen System, Biotech SL, Barcelona, Spain), using and 223 amplitude of 30-55\%, a power of $500 \mathrm{~W}$, and a frequency of 20224 $\mathrm{kHz}$. The sonication probe was placed in a $100 \mathrm{~mL}$ glass beaker at 225 a constant depth, $1.5 \mathrm{~cm}$ above the container bottom.

226

2.4. Vesicle Characterization. 2.4.1. Vesicle Size. The Z- 227 average size and PDI of vesicles were determined by dynamic 228 light scattering (DSL) using a Zetasizer Nano ZS system 229 (Malvern Instruments Ltd., Malvern, U.K.). Three independent 230 samples were taken from each formulation, and measurements 231 

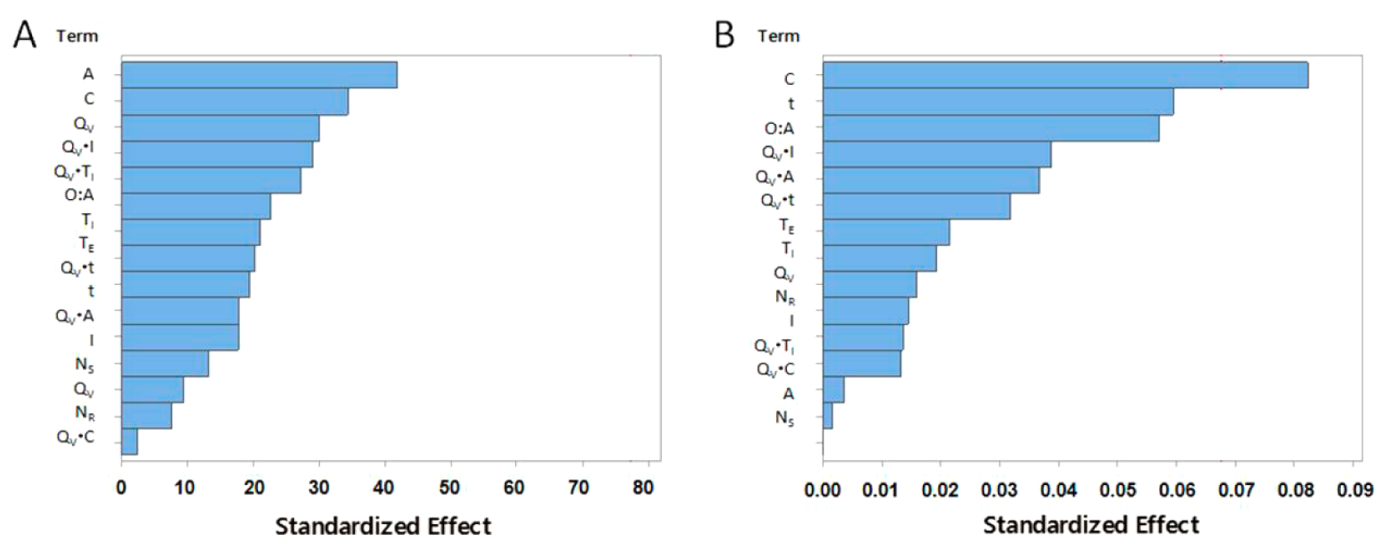

Figure 1. Pareto chart of the standardized effects of independent variables (factors) on the (A) Z-average size and (B) PDI of PC liposomes for the Plackett-Burman fractional factorial design.

232 were performed three times at room temperature without 233 dilution.

$234 \quad$ 2.4.2. Vesicle Morphology. Morphological analysis of vesicles 235 was carried out by negative staining transmission electron 236 microscopy (NS-TEM), using a JEOL-2000 Ex II transmission 237 electron microscope (Tokyo, Japan). A sample drop was placed 238 on a carbon-coated copper grid, and excess sample was removed 239 with filter paper. Then, a drop of $2 \%(\mathrm{w} / \mathrm{v})$ phosphotungstic acid 240 (PTA) solution was applied to the carbon grid and allowed to 241 stand for $1 \mathrm{~min}$. Once the excess staining agent had been 242 removed with filter paper, the sample was air-dried, and the thin 243 film of stained and fixed vesicles was observed with the 244 transmission electron microscope.

245 2.4.3. Vesicle Stability. Vesicle stability was determined by 246 measuring backscattering (BS) profiles in a Turbiscan Lab Expert 247 apparatus (Formulaction, L'Union, France) provided with an 248 aging station (Formulaction, L'Union, France).

249 Samples were placed in cylindrical glass test cells, and 250 backscattered light was monitored at $30{ }^{\circ} \mathrm{C}$ as a function of 251 time and cell height every $2 \mathrm{~h}$ for 7 days.

252 The optical reading head scans the sample in the cell, 253 providing BS data every $40 \mu \mathrm{m}$ in percentages relative to 254 standards as a function of the sample height (in millimeters). 255 These profiles build up a macroscopic fingerprint of the sample at 256 a given time, providing useful information about changes in the 257 size distribution or appearance of a creaming layer or a 258 clarification front with time. ${ }^{3,37,38}$

259 2.4.4. Encapsulation Efficiency (EE). EE also provides useful 260 information related to the stability of the vesicle membrane. 261 Hydrophilic compounds are entrapped in aqueous compart262 ments between bilayers, whereas lipophilic compounds are 263 preferentially located within the surfactant or lipid bilayer. ${ }^{39}$ 264 Substances such as drugs, bioactive compounds, dyes, and 265 nanomaterials incorporated into vesicles can also affect the 266 morphology and stability of the final dispersion.

267 For the purpose of determining EEs, Sudan Red 7B and 268 vitamin $\mathrm{D}_{3}$ (hydrophobic compounds) were encapsulated in the 269 two different formulations.

270 Each compound was analyzed by reverse-phase high-perform271 ance liquid chromatography (RP-HPLC) (HP series 1100 272 chromatograph, Hewlett-Packard, Palo Alto, CA). Before RP273 HPLC analysis could be performed, the nonencapsulated 274 compound had to be removed by passing the sample through a 275 Sephadex G-25 column (GE Healthcare Life Sciences, 276 Wauwatosa, WI). Then, both filtered and nonfiltered samples 277 were diluted 1:10 (v/v) with methanol to facilitate vesicle rupture and to extract the encapsulated compound. EE was 278 calculated according to the equation

$$
\mathrm{EE}(\%)=\frac{\text { peak area of filtered sample }}{\text { peak area of unfiltered sample }} \times 100
$$

The RP-HPLC system was equipped with an HP G1315A UV/ 281 vis absorbance detector (Agilent Technologies, Palo Alto, CA). 282 The column was a Zorbax Eclipse Plus C18 column with a 283 particle size of $5 \mu \mathrm{m}, 4.6 \mathrm{~mm} \times 150 \mathrm{~mm}$ (Agilent Technologies, 284 Palo Alto, CA). The mobile phase consisted of a mixture of (A) 285 $100 \%$ Milli-Q-water and (B) $100 \%$ methanol with gradient 286 elution at $0.8 \mathrm{~mL} / \mathrm{min}$. The step gradient started with a mobile 287 phase of $80 \%$ A, running $100 \%$ mobile phase B starting in minute 288 5 for $10 \mathrm{~min}$. Mobile phase B was fed for $2 \mathrm{~min}$ after each 289 injection to prepare the column for the next sample. The 290 separation was carried out at $30{ }^{\circ} \mathrm{C}$. Different wavelengths were 291 used for the UV/vis detector, namely, $533 \mathrm{~nm}$ for Sudan Red 7B 292 and $270 \mathrm{~nm}$ for vitamin $\mathrm{D}_{3}$

293

\section{RESULTS AND DISCUSSION}

3.1. Effects of Variables on Morphological Character- 294 istics. The responses ( $Z$-average size and PDI) of each batch 295 from P-B design were measured by dynamic light scattering 296 (DLS). The relative importance of the main effects on the Z- 297 average size and PDI of PC liposomes are shown in the Pareto 298 chart given in Figure 1.

Researchers must be aware of the confusion of main effects 300 with two-factor interactions in this type of design (resolution III), 301 where the alias structure is too complex. However, we decided to 302 use the initial Plackett-Burman design only for screening 303 purposes and selection of the main factors from the Pareto chart, 304 as is usually accepted. Effects were selected by applying the 305 hierarchical ordering principle, known sometimes as the sparsity- 306 of-effects principle, where higher-order effects (three- or four- 307 way interactions) are sacrificed to study lower-order effects 308 (main effects first and two-way interactions next). This principle 309 suggests that priority should be given to the estimation of lower- 310 order effects, especially when resources (time and money) are 311 scarce. This postulate is an empirical principle whose validity has 312 been confirmed by the analysis of many real experiments. 313

According to these data, the most important variables for both 314 responses are the organic/aqueous phase volume ratio, the (final 315 aqueous-phase) phospholipid concentration, and the sonication 316 amplitude. These results are in good agreement with previous 317 studies carried out by Kremer et al., ${ }^{40}$ who evaluated the effects of 318 

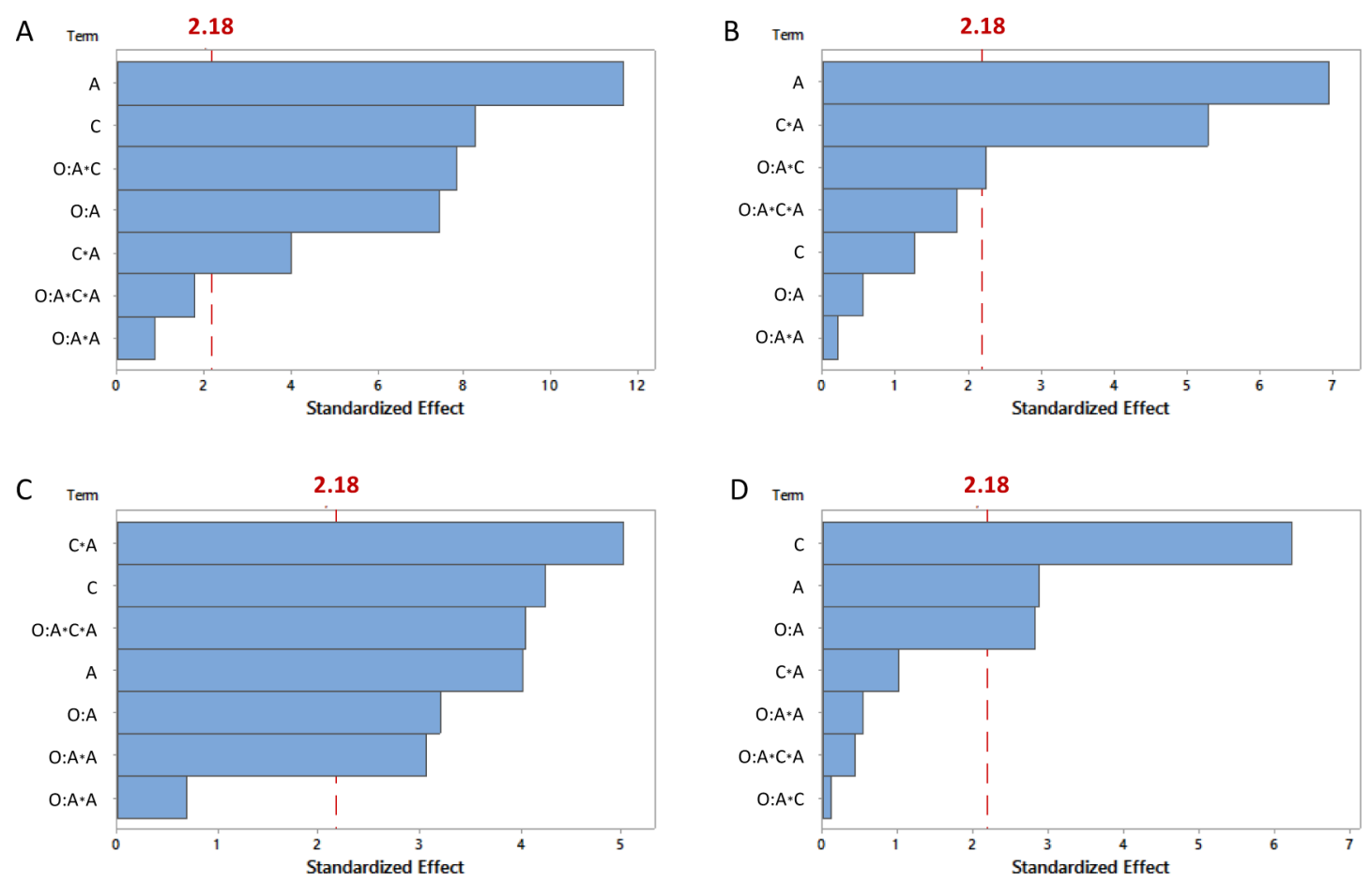

Figure 2. Pareto chart of the standardized effects of independent variables (factors) on the (A,C) Z-average size and (B,D) PDI of (A,B) PC liposomes and $(C, D)$ S60:Cho niosomes $(1: 0.5, w / w)$ for the $2^{3}$ full factorial design.

319 some preparation variables on the size and polydispersity of 320 liposomes made from two different natural phosphatidylcholines. 321 Their experimental results showed that the most important 322 factor in the final size of the liposomes was the lipid 323 concentration in the alcohol injected into the buffer solution. 324 This factor corresponds to the interaction of the lipid amount 325 and the flow rate of organic solvent injected, two factors present 326 in the Pareto chart in Figure 1. The same explanation was 327 postulated by other authors, ${ }^{8,41,42}$ confirming that the lipid 328 concentration clearly affects the liposome size. This factor was 329 found to be the most relevant one for controlling morphological 330 characteristics of phosphatidylcholine liposomes. Szoka ${ }^{43}$ found 331 that stirring, ionic strength, and temperature of the aqueous 332 phase could also contribute to the final size, but the effects of 333 these factors were smaller than those observed for lipid 334 concentration, organic/aqueous phase ratio, and chemical nature 335 of the organic solvent (a parameter not included in our study). 336 Therefore, the experimental results in Figure 1 confirm the 337 previously reported observations. ${ }^{43}$

338 The ethanol injection method is usually chosen because it 339 avoids the sonication step, which is needed in several other 340 methods of liposome preparation, such as the thin-film hydration 341 method. Preliminary experiments (data not shown) indicated 342 that sonication is a crucial step for reducing the size of both 343 liposomes and niosomes. Alternatively, small vesicles can be 344 produced without sonication by using low concentrations of 345 lipids/surfactants, but with low yield. This is why we decided to 346 include this step as a factor in the present study.

347 3.2. PC Liposomes. The first three main effects from the 348 Pareto chart obtained for the $\mathrm{P}-\mathrm{B}$ design were selected for the $2^{3}$ 349 full factorial design. The ANOVA results for $Z$-average size and 350 PDI values are listed in Tables S1 and S2 (Supporting 351 Information), respectively, whereas the corresponding Pareto 352 chart is shown in Figure 2. Mean sizes in the range of 55-156 nm 353 with PDI values between 0.173 and 0.378 were obtained for PC liposomes (with standard deviations ranging from 0.304 to 4.40354 $\mathrm{nm}$ for size and from 0.003 to 0.053 for PDI). Similar size ranges 355 were also obtained using the EIM in other previously reported 356 studies. $22,27,41,43,44$

The normality, variance homogeneity, and randomness 358 assumptions were tested with a normal probability plot, 359 frequency histogram, and residuals versus fits and residuals 360 versus order plots, respectively (Supporting Information, Figure 361 S2).

No clear aberrant tendencies were observed, because the 363 residuals tended to form a line, no typical cornet pattern was 364 observed, and no time-based pattern was detected. Only some 365 outlier values were detected (Cook's distance and DFITS values 366 are given in Table S3 of the Supporting Information). 367

The ANOVA results allowed for an analysis of the 368 contributions of the effects of the independent variables on the 369 response function (mean size of PC liposomes). In this case, 370 significant two-way interactions were identified: $(\mathrm{O} / \mathrm{A}) \times \mathrm{C}$ and 371 $C \times A$ (see Figure 3). Larger sizes are reached when the organic $372 \mathrm{fz}$ solution has a higher lipid concentration (more than $20 \mathrm{~g} / \mathrm{L}$ ). On 373 the other hand, the $C \times A$ interaction reveals that the degree of 374 size reduction upon application of a higher amplitude depends 375 on the total lipid concentration present in the medium (referred 376 to the final volume of the dispersion).

All of the main effects are significant $(p<0.05)$, with a positive 378 effect on the mean size (a higher response value with an increase 379 in the factor level) for the total lipid concentration and a negative 380 effect (a lower response value with a decrease in factor level) for 381 the organic/aqueous phase volume ratio and the sonication 382 amplitude.

These effects can be explained according to a previously 384 reported vesicle formation model. ${ }^{45-47}$ This model relies on the 385 formation of vesicles through intermediate structures, such as 386 phospholipid bilayer fragments and sheet-like micelles. These 387 
A

B
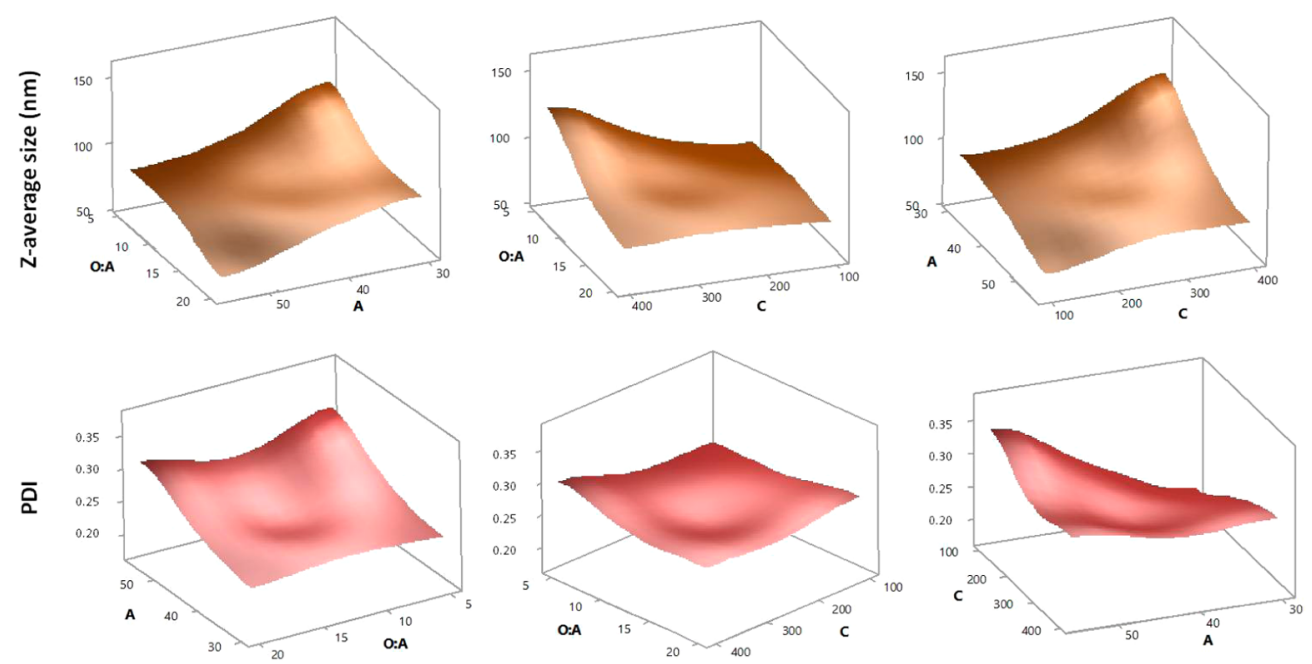

C
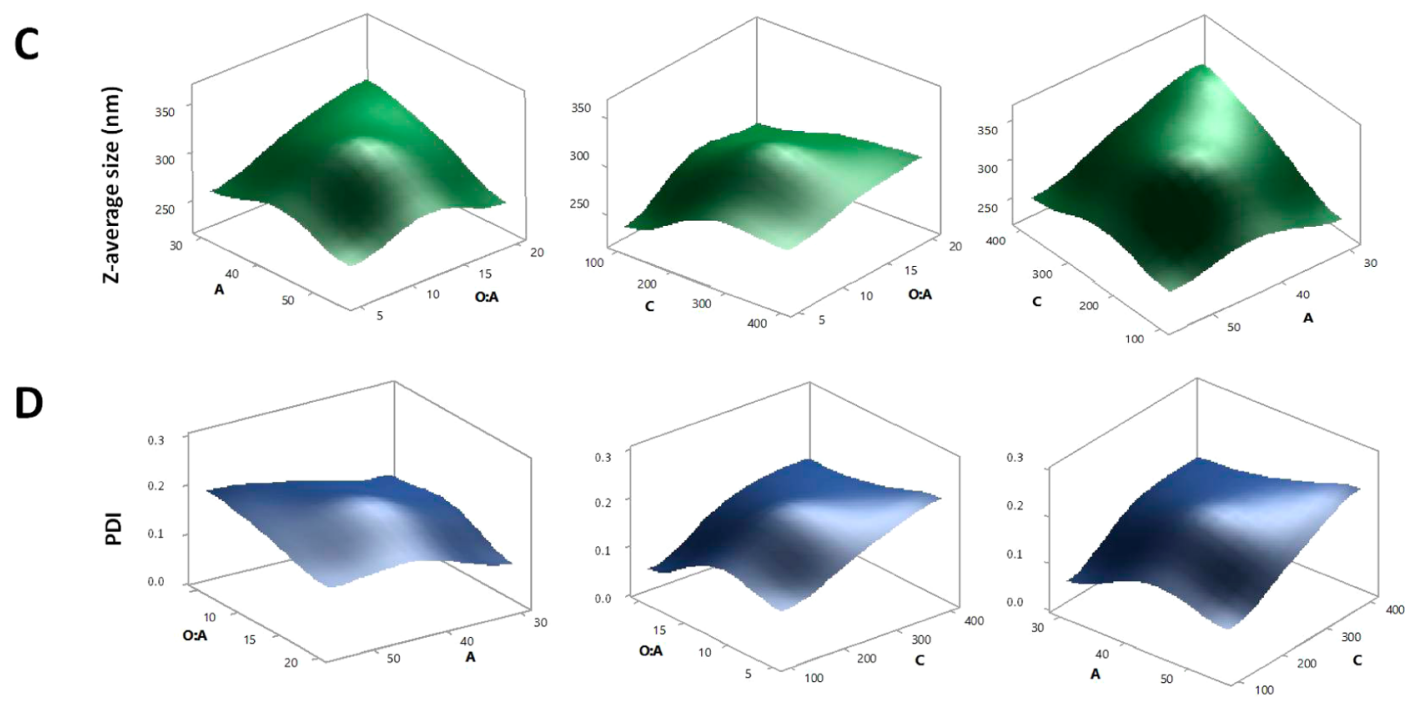

Figure 3. Three-dimensional (3D) response surface plots for the factors $\mathrm{O} / \mathrm{A}$ (organic/aqueous phase volume ratio), $\mathrm{C}$ (lipid or surfactant/stabilizer concentration, $\mathrm{g} / \mathrm{L}$ ), and A (sonication amplitude, \%) for the (A,C) $Z$-average size and (B,D) PDI of (A,B) PC liposomes and (C,D) S60:Cho niosomes $(1: 0.5, \mathrm{w} / \mathrm{w})$.

388 intermediates are the result of amphiphilic self-assembly because 389 of their characteristic physicochemical properties. ${ }^{48}$

390 During the injection of ethanol droplets into the aqueous 391 phase, lipid reorganization inside these dispersed droplets to 392 form bilayers is favored by the fact that lipids energetically prefer 393 a parallel molecular arrangement. ${ }^{45}$ These planar structures give 394 rise to closed vesicles when their size induces enough surface 395 tension to close the structure and minimize the bending energy. 396 The sizes of these intermediates depend directly on the 397 number of lipid molecules (concentration) and the dispersion 398 degree (solubilization) in the organic phase. It is obvious from 399 the previous assessment that higher concentrations of lipids in 400 the droplets will form higher membrane fragments, as our 401 experimental results and previous observations confirm. ${ }^{8,40-42}$

402 It is also important to know how easily lipid droplets are 403 dispersed, as well as their size and homogeneity. Lipids of higher 404 solubility will then form smaller lipid droplets and, consequently, 405 shorter membrane fragments (and ultimately tiny vesicles). ${ }^{40}$ 406 This explains, in a simplified way, why higher organic/aqueous 407 phase ratios yield smaller liposomes.

408 The negative effect of the sonication amplitude is explained by 409 vesicle rupture, which takes place when an excess of energy is applied to vesicles during the sonication process as a result of the 410 effect of induced cavitation. ${ }^{49,50}$ The final effect of ultrasounds 411 can be controlled by varying the input power, ultrasound 412 frequency, sonication time, and probe depth into the container. 413 As frequency increases, liposomes of smaller size are produced as 414 a result of stronger acoustic cavitation events. This assumption 415 was confirmed by our results, in accordance with previous 416 studies. ${ }^{49,50}$ It is important to point out that, to minimize the 417 effects of variations in the probe depth, this factor was kept 418 constant at $1.5 \mathrm{~cm}$ above the container bottom.

419

Another aspect to be taken into consideration is the effect of 420 sonication time. It was reported by Silva et al. ${ }^{49}$ that sonication 421 time plays an important role in decreasing vesicle size, although 422 they observed that this effect reached a plateau at about $21 \mathrm{~min} .423$ Our P-B design revealed a positive effect of sonication time on 424 the $Z$-average size (from 15 to $30 \mathrm{~min}$ ), although it was weaker 425 than the effects of the other variables selected for the $2^{3}$ full 426 factorial design (especially sonication amplitude). A similar 427 influence was observed for the PDI response, but with a stronger 428 effect. We preferred to select sonication amplitude instead of 429 sonication time because one of the goals of controlling factors is 430 to obtain a narrow size distribution. 
Table 3. Estimated Coded Coefficients for the Considered Effects on the Z-Average Size and PDI of PC Liposomes and S60:Cho Niosomes $(1: 0.5, \mathrm{w} / \mathrm{w})$

\begin{tabular}{|c|c|c|c|c|c|c|c|c|c|}
\hline \multirow[b]{2}{*}{ response } & \multicolumn{9}{|c|}{ coefficients $^{a}$} \\
\hline & constant & $X_{1}$ & $X_{2}$ & $X_{3}$ & $X_{1} X_{2}$ & $X_{1} X_{3}$ & $X_{2} X_{3}$ & $X_{1} X_{2} X_{3}$ & $R^{2}$ \\
\hline \multicolumn{10}{|c|}{ Z-Average Size } \\
\hline liposome $\left(Y_{1}\right)$ & 89.68 & -11.14 & 12.40 & -17.50 & -11.75 & - & -5.97 & - & 96.69 \\
\hline niosome $\left(Y_{3}\right)$ & 269.82 & 12.72 & 16.87 & -15.94 & - & -12.15 & -19.92 & -16.04 & 91.27 \\
\hline \multicolumn{10}{|c|}{ PDI } \\
\hline liposome $\left(Y_{2}\right)$ & 0.280 & - & - & 0.038 & -0.012 & - & -0.029 & - & 89.35 \\
\hline niosome $\left(Y_{4}\right)$ & 0.136 & -0.026 & 0.057 & 0.026 & - & - & - & - & 84.73 \\
\hline
\end{tabular}

${ }^{a} X_{1}$, organic/aqueous phase volume ratio; $X_{2}$, PC or S60:Cho concentration $(\mathrm{g} / \mathrm{L}) ; X_{3}$, sonication amplitude (\%).

432 As the design included a center point with several repetitions $433(n=5)$, the presence of curvature in the response variables could 434 be tested (Figure 3). Because curvature seemed to be significant $435(p<0.05)$, a term involving center point $(\mathrm{Ct} \mathrm{Pt})$ was included in 436 the equations for its estimation.

437 With all of this information about the effects and their 438 estimated coefficients, the following equation $\left(R^{2}=96.69 \%\right)$ for 439 the $Z$-average size value of PC liposomes $\left(Y_{1}\right)$ was generated

$$
\begin{aligned}
Y_{1} & =62.8+2.55(\mathrm{O} / \mathrm{A})+0.449 C-0.185 A \\
& -0.0185(\mathrm{O} / \mathrm{A}) \times C-0.00555 C \times A-9.26(\mathrm{Ct} \mathrm{Pt})
\end{aligned}
$$

440

441 Different behavior was observed regarding PDI, which was 442 strongly affected by the sonication amplitude as the only 443 significant main effect and its interaction with the total lipid 444 amount. The $\mathrm{O} / \mathrm{A} \times \mathrm{C}$ interaction was also detected, but with a 445 lower effect on the PDI response.

446 To understand the $C \times A$ interaction, it is important to take 447 into account the effect of the sonication amplitude as the main 448 effect. An increase in this factor leads to a less monodisperse size 449 distribution, that is, higher PDI values. However, according to 450 the interaction, this response depends highly on the total amount 451 of lipids present in the sample. At a low level of the lipid amount, 452 the reduction in size is more effective (as previously mentioned), 453 but the size distribution is large. However, at a high level of the 454 lipid amount, this enlargement of the size distribution is 455 significantly lower.

456 Curvature in the response was also tested, again revealing a 457 significant presence $(p<0.05)$. For the PDI response $\left(Y_{2}\right)$, the 458 following equation with an $R^{2}$ value of $89.35 \%$ was obtained

459

$$
\begin{aligned}
Y_{2} & =-0.160+0.00939 A-0.0000420(\mathrm{O} / \mathrm{A}) \times C \\
& -0.0000250 C \times A-0.0425(\mathrm{Ct} \mathrm{Pt})
\end{aligned}
$$

460 These equations were formulated with uncoded coefficients, 461 making it easier to use them to predict selected target size and 462 PDI values.

463 3.3. S60:Cho Niosomes. To investigate whether the 464 selected factors in the $\mathrm{P}-\mathrm{B}$ design for PC liposomes (a reference 465 model for vesicular systems) produced similar effects with other 466 different formulations, the same $2^{3}$ full factorial design using 467 center-point replicates was performed for a typical niosome 468 formulation, in this particular case, S60:Cho niosomes (1:0.5, w/ $469 \mathrm{w}$ ). The main variables were the organic/aqueous phase volume 470 ratio $(\mathrm{O} / \mathrm{A})$, the total concentration of surfactant and stabilizer $471(C)$, and the sonication amplitude $(A)$.

472 The ANOVA results for $Z$-average size and PDI values are 473 listed in Table S1 (Supporting Information), and the 474 corresponding Pareto chart and three-dimensional surface plot are shown in Figures 2 and 3, respectively. Mean sizes in the 475 range of 224-362 nm with PDI values between 0.032 and 0.291476 were obtained for S60:Cho niosomes (with standard deviations 477 ranging from 1.05 to $7.28 \mathrm{~nm}$ for size and from 0.009 to 0.052 for 478 PDI). Similar size and PDI ranges were reported for niosomes 479 prepared by the EIM using Span 60 as the membrane 480 component. $^{17}$

Two-way interactions $(\mathrm{O} / \mathrm{A} \times A, C \times A)$ and a three-way 482 interaction $(\mathrm{O} / \mathrm{A} \times \mathrm{C} \times \mathrm{A})$ were detected, with sonication 483 amplitude $(A)$ as the common factor in these interactions (see 484 Figure 2C). Therefore, it can be postulated that sonication 485 amplitude is the key factor in the niosome size response. The 486 response depends on both the $\mathrm{O} / \mathrm{A}$ and $C$ factor levels, with a 487 higher interaction between the sonication amplitude and the 488 total amount of membrane components. Differences in the 489 magnitude of the coefficient of this factor between liposomes and 490 niosomes can be attributed to the initial size before sonication 491 (smaller for liposomes) and vesicle stability. ${ }^{50}$

The three main effects are significant, but in contrast to the 493 case for liposomes, the organic/aqueous phase volume ratio $(\mathrm{O} / 494$ A) shows a positive effect on niosome size. This behavior could 495 be due to different molecular features of the surfactant and 496 stabilizer that result in different interactions with the organic 497 phase and, therefore, poor or insufficient solubility.

498

The other two variables $(C, A)$ have effects similar to those 499 described above for liposomes. Therefore, the same explanation 500 regarding surfactant concentration and sonication amplitude can 501 be applied here to justify their effects on niosome size. In this 502 case, the stronger effect of $C$ is explained by the influence of 503 cholesterol on the final size of vesicles, as reported by Padamwar 504 and Pokharkar. ${ }^{8}$

Once again, curvature was detected for the $Z$-average size 506 response. The following equation was obtained to model this 507 case, with an adjusted correlation coefficient $\left(R^{2}\right)$ of $91.27 \% \quad 508$

$$
\begin{aligned}
Y_{3} & =236.9-4.31(\mathrm{O} / \mathrm{A})-0.012 C-0.56 A \\
& +0.0461(\mathrm{O} / \mathrm{A}) \times C+0.00363 C \times A \\
& -0.00114(\mathrm{O} / \mathrm{A}) \times C \times A+44.00(\mathrm{CtPt})
\end{aligned}
$$

On the other hand, a completely different behavior was observed 510 regarding the PDI response. Only the three main effects (O/A, C, 511 $A$ ) were found to be significant, and no interactions were found. 512 Two positive effects on the niosome PDI were detected: 513 surfactant/stabilizer concentration and sonication amplitude. In 514 this case, the total concentration of membrane components 515 seemed to have an important role in the vesicle size distribution, 516 as can be seen in the correspondent Pareto chart (Figure 2). This 517 observation once again can be attributed to the solubilization of 518 membrane components in the organic phase. Higher concen- 519 


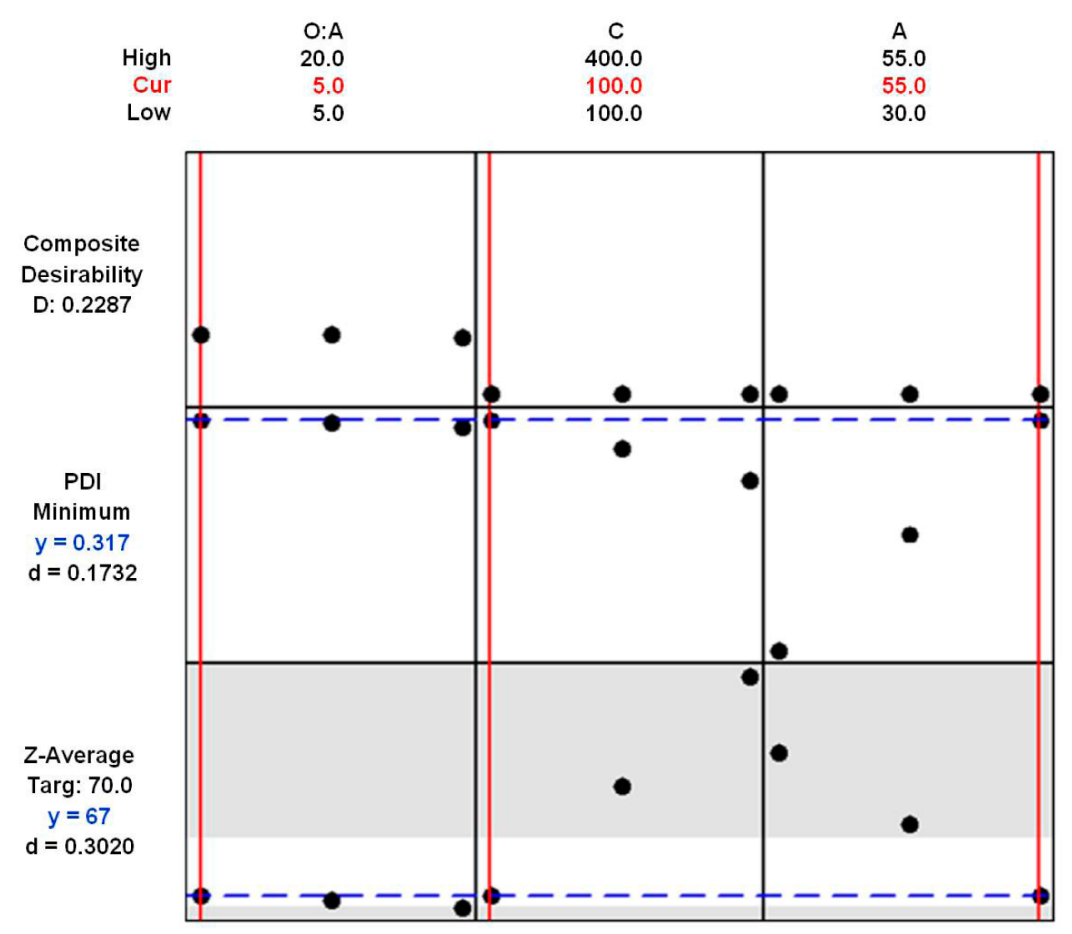

Parameters

$\begin{array}{llrrrrr}\text { Response } & \text { Goal } & \text { Lower } & \text { Target } & \text { Upper } & \text { Weight } & \text { Importance } \\ \text { Z-Average PC-Liposomes } & \text { Target } & 65 & 70.000 & 75.000 & 1 & 1 \\ \text { PDI PC-Liposomes } & \text { Minimum } & & 0.173 & 0.378 & 1 & 1\end{array}$

Solution

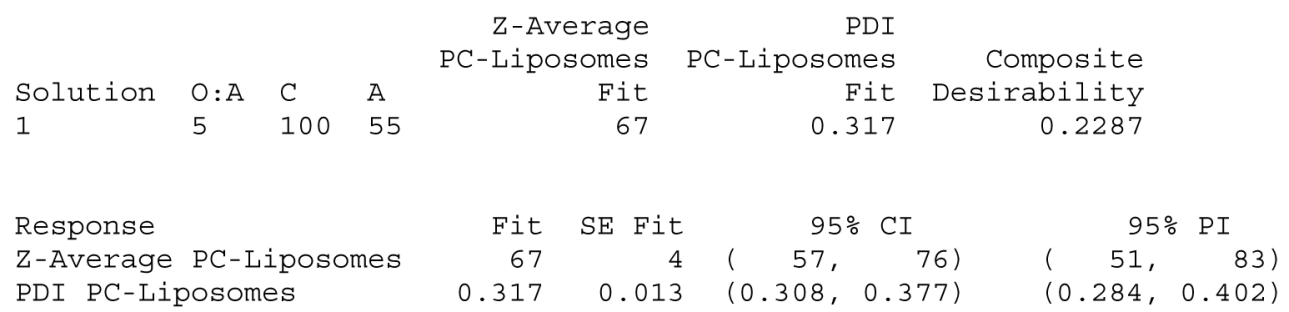

Figure 4. Optimization plot and values of individual $(d)$ and composite $(D)$ desirability provided by the response optimizer (Minitab, version 17$)$ for an example of size-tuned PC liposome (desired size $=70 \mathrm{~nm}$, with a minimum PDI value).

520 trations of these components require better solubilization in 521 dispersed droplets to reach small membrane fragments.

522 It is important to note that some combinations of factors 523 yielded narrow size distributions, namely, PDI $\leq 0.100$, a value 524 frequently obtained by other preparation methods, such as 525 microfluidic hydrodynamic focusing ${ }^{51}$ also using S60:Cho as the 526 formulation.

527 A negative effect was detected for the organic/aqueous phase 528 volume ratio $(\mathrm{O} / \mathrm{A})$. As the final concentration of ethanol 529 increased during the injection process, a smaller size distribution 530 was obtained. As previously mentioned, no interaction between 531 this factor and the total concentration of membrane components 532 was observed.

533 The following equation with an $R^{2}$ value of $84.73 \%$ was 534 obtained for the niosome PDI model response $\left(Y_{4}\right)$

$$
\begin{aligned}
Y_{4} & =0.053-0.00392(\mathrm{O} / \mathrm{A})+0.000039 \mathrm{C}+0.00067 \mathrm{~A} \\
& +0.0597(\mathrm{Ct} \mathrm{Pt})
\end{aligned}
$$

The estimated coded coefficients for the considered effects on 536 the $Z$-average sizes and PDIs of PC liposomes and S60:Cho 537 niosomes are listed in Table 3, as a summary of the factors' $538 \mathrm{t} 3$ influence. Coded coefficients were used to maintain the 539 orthogonality of the designs and to allow for a direct comparison 540 between coefficients.

3.4. Vesicle Characterization. Size-tuned vesicles were 542 prepared under selected operating conditions by applying the 543 models obtained from the experimental design (eqs 2-5) and 544 the assistance of the response optimizer and response predictor 545 in Minitab statistical software (version 17). These tools can be 546 applied to the simultaneous optimization of several responses 547 only when the same set of factors are studied separately, because 548 a common experimental region is needed.

The operating conditions were selected to prepare PC 550 liposomes with a mean size of $70 \mathrm{~nm}$ and the minimum PDI 551 value (predicted values of $Y_{1}=67 \pm 4$ and $Y_{2}=0.317 \pm 0.013$ ) 552 and S60-Cho niosomes with a mean size of $240 \mathrm{~nm}$ and the 553 


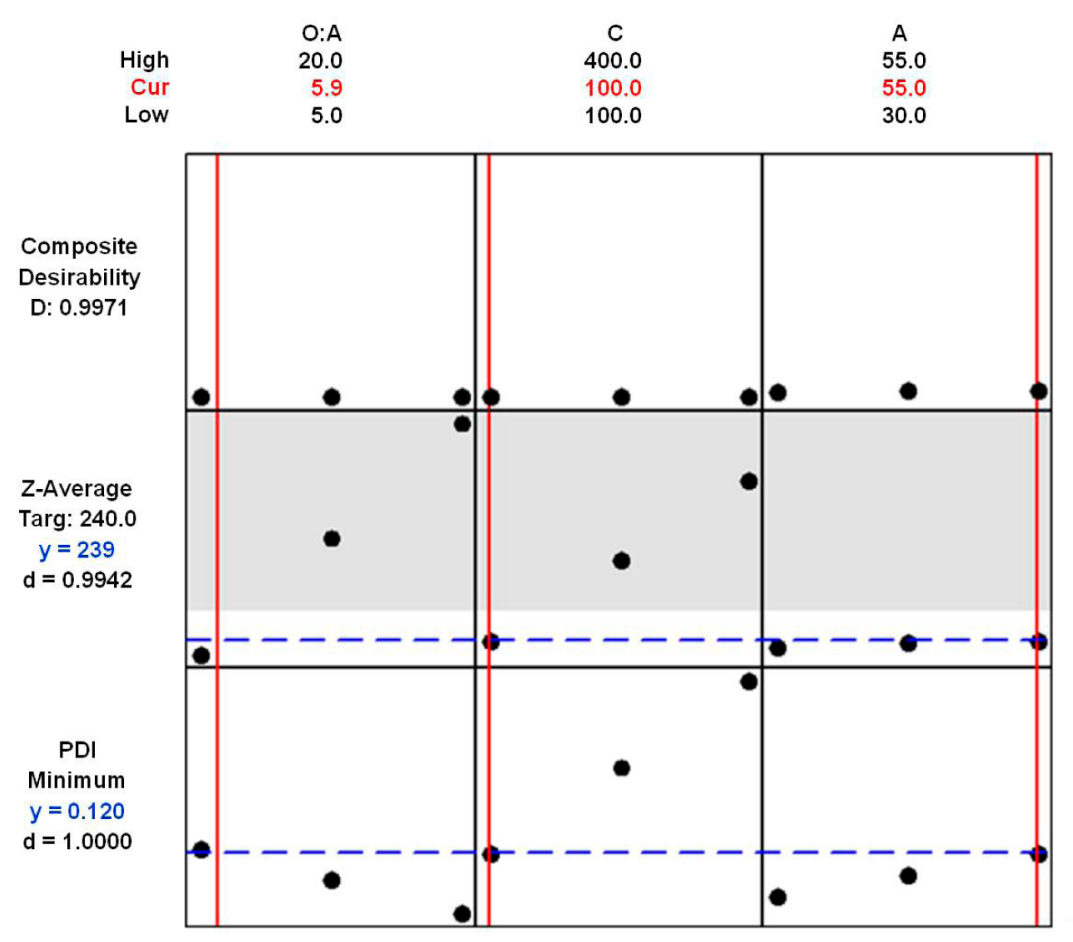

Parameters

$\begin{array}{llrrrr}\text { Response } & \text { Goal } & \text { Lower } & \text { Target } & \text { Upper Weight } \\ \text { Importance } & & & & & \\ \text { Z-Average S60:Cho Niosomes } & \text { Target } & 235 & 240.000 & 245.000 & 1 \\ 1 & \text { Minimum } & & 0.120 & 0.291 & 1 \\ \text { PDI S60:Cho Niosomes } & \text { Mind }\end{array}$

1

Solutions

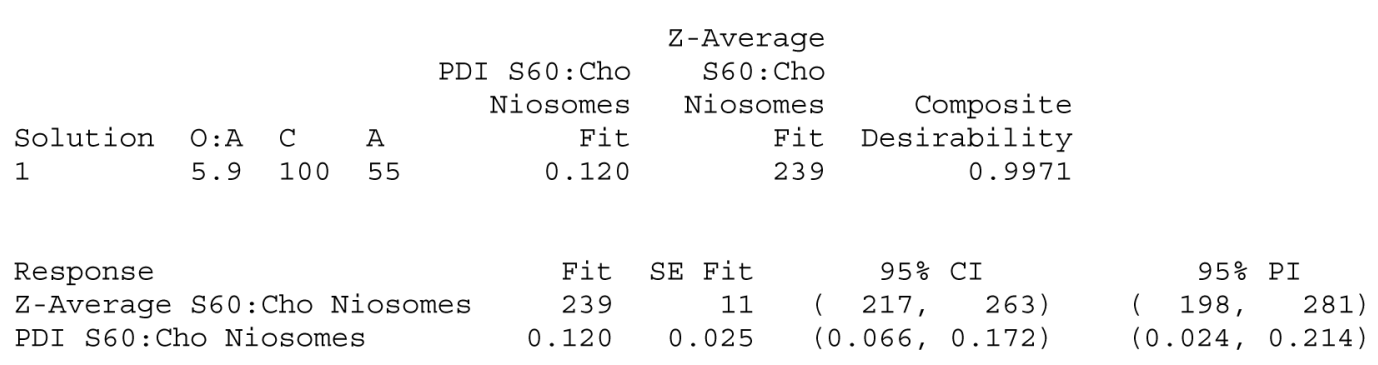

Figure 5. Optimization plot and values of individual $(d)$ and composite $(D)$ desirability provided by the response optimizer (Minitab, version 17$)$ for an example of size-tuned S60:Cho niosome $(1: 0.5 \mathrm{w} / \mathrm{w})$ (desired size $=240 \mathrm{~nm}$, with a minimum PDI).

554 minimum PDI value (predicted values of $Y_{3}=239 \pm 11$ and $Y_{4}=$ $5550.120 \pm 0.025)$. These sizes and PDI values were selected only as 556 an example. The factor output values were $\mathrm{O} / \mathrm{A}=5: 50, \mathrm{C}=2 \mathrm{~g} /$ $557 \mathrm{~L}$, and $A=55 \%$ for the liposomes and $\mathrm{O} / \mathrm{A}=5.9: 50, C=2 \mathrm{~g} / \mathrm{L}$, 558 and $A=55 \%$ for the niosomes. Figures 4 and 5 show optimization 559 plots and values of individual and composite desirability for size560 tuned liposomes and size-tuned niosomes, respectively.

561 The experimental results showed that the models obtained 562 with the experimental design were accurate, because mean sizes 563 of $69 \pm 0.5 \mathrm{~nm}(\mathrm{PDI}=0.245 \pm 0.005)$ and $233 \pm 3 \mathrm{~nm}(\mathrm{PDI}=$ $5640.112 \pm 0.004)$ were obtained for the PC liposomes and S60:Cho 565 niosomes, respectively. The relative error was low for the 566 experimental results regarding mean size $\left(3 \%\right.$ for $Y_{1}$ and $\left.Y_{3}\right)$ but 567 higher for the size distributions ( $22 \%$ for $Y_{2}$ and $7 \%$ for $Y_{4}$ ).

568 The sizes and morphologies of the vesicles were investigated f6 vesicles, as a result of the interactions of the electron beam with 570 PTA, which produces a selective deposit of metal ions that 571 enhances morphological details. The micrographs show spherical 572 structures of approximately $80 \mathrm{~nm}$ for the liposomes (Figure 6C) 573 and about $250 \mathrm{~nm}$ for the niosomes (Figure 6D). These values 574 agree with the DLS measurements.

575

Figure $6 \mathrm{D}$ shows clusters of niosomes that are all similar in 576 size. Aggregation arose during the drying step prior to TEM 577 measurements, because no flocculation phenomena were 578 monitored with the Turbiscan apparatus.

Slight differences were noticed in the zeta potential measure- 580 ments, exhibiting low values for both types of vesicles. Niosomes 581 had values of about $-16.8 \pm 0.7 \mathrm{mV}$, whereas the liposomes had 582 values of $-6.9 \pm 0.3 \mathrm{mV}$. This small value for the liposomes could 583 be due to neutralization of the negative charge from the 584 

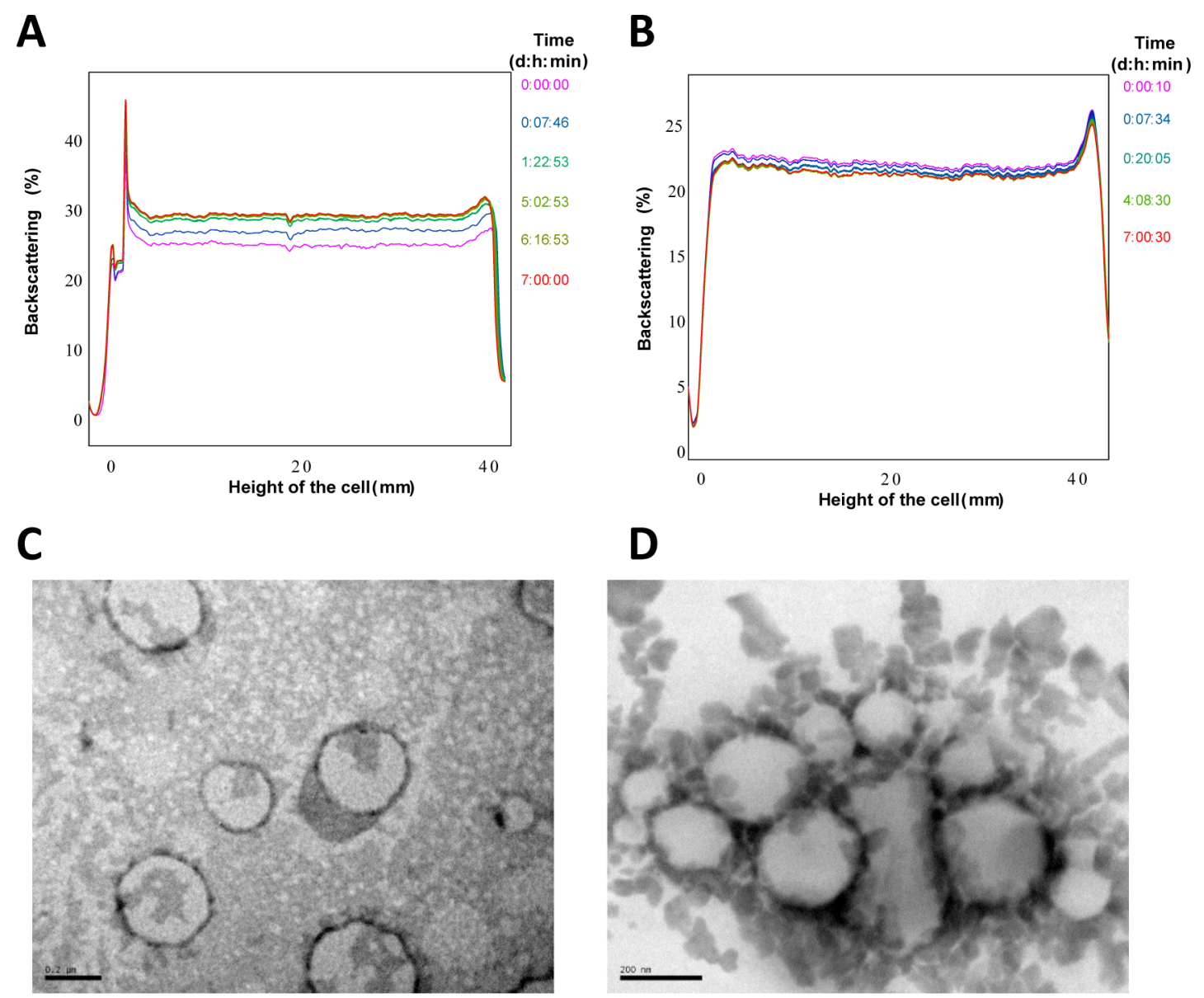

D

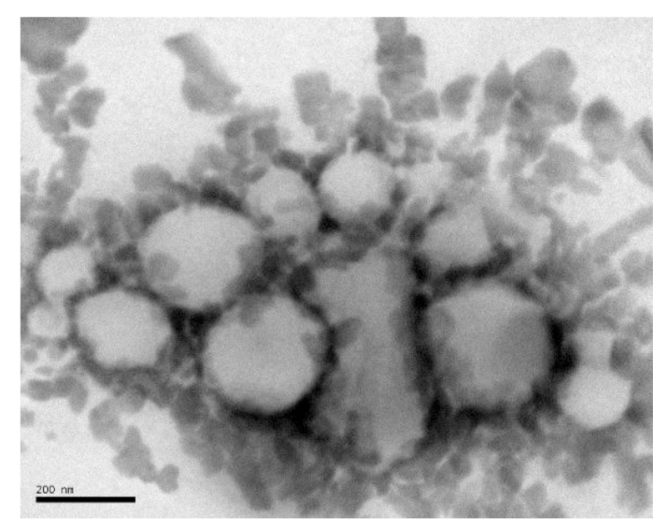

Figure 6. (A,B) BS profiles and (C,D) TEM micrographs of empty vesicles designed with a controlled size and PDI values by applying the models obtained from experimental design: $(A, C)$ PC liposomes and (B,D) S60:Cho niosomes (1:0.5, w/w).

585 phosphate groups by sodium cations present in the medium 586 (from sodium chloride in the PB solution).

587 The formulated vesicles exhibited a high stability after 1 week 588 of monitoring time. BS profiles obtained for the PC liposomes 589 are given in Figure 6, where a variation of $4.5 \%$ in the middle part 590 of the cell (from 10 to $30 \mathrm{~mm}$ ) is noticed. A simultaneous slight 591 clarification process was observed in the middle and top parts of 592 the cell in the corresponding transmission profile (results not 593 shown). This was promoted by some movement of the PC 594 liposomes toward the bottom of the cell, resulting in a slight 595 increase in BS (sedimentation). However, this was a reversible 596 process, caused by differences in concentration, with the sample 597 remaining stable and maintaining its initial properties (size and 598 PDI). The vesicles were again characterized after gentle agitation 599 of the cell at the end of the monitoring time with analogous 600 results.

601 For the S60:Cho niosomes (Figure 6B), the BS profile 602 remained nearly constant (variations of approximately $0.5 \%$ ) 603 with time, showing high stability. Some variation was also 604 observed in the transmission profile all along the cell height, 605 because the sample was not translucent.

606 3.4.1. Encapsulation Efficiency (EE). Vesicles containing ${ }_{607}$ Sudan Red 7B and vitamin $\mathrm{D}_{3}$ as model compounds (both 608 lipophilic) were also prepared and characterized. No differences 609 were observed regarding mean size and PDI values or TEM, zeta 610 potential, or Turbiscan measurements, meaning that the 611 entrapped compounds did not affect the vesicle's behavior.
High EE values were obtained for both Sudan Red 7B and 612 vitamin $\mathrm{D}_{3}$, as expected taking into account their hydrophobic 613 character. EE values up to $90.1 \%$ and $88.0 \%$ were obtained for 614 Sudan Red 7B encapsulated in PC liposomes and S60:Cho 615 niosomes, respectively. Experiments carried out with vitamin $\mathrm{D}_{3} 616$ led to EE values of $99.2 \%$ for PC liposomes and $73.9 \%$ for 617 S60:Cho niosomes. These results are in good agreement with 618 those of previous studies, where compounds with similar 619 chemical properties were encapsulated. ${ }^{12,13,27}$

620

\section{CONCLUSIONS}

In this work, an adequate approximation using DoE was applied 621 to study the influence of experimental factors of the EIM on the 622 mean size and size distribution of PC liposomes and S60:Cho 623 niosomes $(1: 0.5, \mathrm{w} / \mathrm{w})$.

An initial screening design enabled a reduction of the number 625 of variables. This was a necessary step before carrying out a full 626 factorial design. Finally, response models were applied to prepare 627 selected size-tuned nanovesicles, which were characterized from 628 a stability point of view.

629

This was achieved with a low number of experiments (58 630 runs). This methodology enabled two different formulations 631 (liposomes and niosomes, the most common types of nano- 632 vesicles) to be studied in a comparative way. Stable liposomes 633 and niosomes of the targeted sizes were successfully prepared 634 with the model equations obtained, with encapsulation 635 efficiencies higher than $73.9 \%$ in all cases for selected 636 hydrophobic compounds. 
638 The most important variables identified by ANOVA were the 639 organic/aqueous phase volume ratio, the (final aqueous-phase) 640 phospholipid concentration, and the sonication amplitude.

641 These results offer new insights into the mechanism and effects 642 of the factors involved in nanovesicle preparation by the EIM, 643 one of the most easily scaled-up methods for preparing vesicles 644 for several fields of interest.

\section{ASSOCIATED CONTENT}

\section{S Supporting Information}

647 The Supporting Information is available free of charge on the 648 ACS Publications website at DOI: 10.1021/acs.iecr.6b01552.

649 ANOVA results for $Z$-average size and PDI of PC 650 liposomes for the $2^{3}$ full factorial design; Cook's distances 651 and DFITS values for each response in the full factorial 652 designs; optimization contour plot for the factors studied 653 in the full factorial design for both responses; testing for 654 normality, variance homogeneity, and randomness 655 assumptions of ANOVA for the full factorial design $656 \quad(\mathrm{PDF})$

\section{AUTHOR INFORMATION}

\section{Corresponding Author}

659 *Tel.: +34 985103509. E-mail: cpazos@uniovi.es.

\section{Notes}

661 The authors declare no competing financial interest.

\section{ACKNOWLEDGMENTS}

663 This work was supported by the Ministerio de Economía y 664 Competitividad (MINECO, Spain), under Grant MINECO-13665 CTQ2013-47396-R. This study was also cofinanced by the 666 Consejería de Educación y Ciencia del Principado de Asturias 667 (ref FC-04-COF-50-MEC, PCTI Asturias 2006-2009, ref 668 EQP06-024, and FC15-GRUPIN14-022). We especially thank 669 Prof. Antonia Salas (University of Oviedo) for her advice with 670 the statistical work.

\section{REFERENCES}

672 (1) Capretto, L.; Carugo, D.; Mazzitelli, S.; Nastruzzi, C.; Zhang, X. 673 Microfluidic and lab-on-a-chip preparation routes for organic nano674 particles and vesicular systems for nanomedicine applications. Adv. Drug 675 Delivery Rev. 2013, 65, 1496-1532.

676 (2) Rongen, H. A. H.; Bult, A.; van Bennekom, W. P. Liposomes and 677 immunoassays. J. Immunol. Methods 1997, 204, 105-133.

678 (3) Pando, D.; Gutiérrez, G.; Coca, J.; Pazos, C. Preparation and 679 characterization of niosomes containing resveratrol. J. Food Eng. 2013, $680117,227-234$.

681 (4) Gómez-Hens, A.; Fernández-Romero, J. M. The role of liposomes 682 in analytical processes. TrAC, Trends Anal. Chem. 2005, 24, 9-19.

683 (5) Edwards, K. A.; Bolduc, O. R.; Baeumner, A. J. Miniaturized 684 bioanalytical systems: enhanced performance through liposomes. Curr. 685 Opin. Chem. Biol. 2012, 16, 444-452.

686 (6) Liu, Q.; Boyd, B. J. Liposomes in biosensors. Analyst 2013, 138, 687 391-409.

688 (7) Canton, I.; Battaglia, G. Endocytosis at the nanoscale. Chem. Soc. 689 Rev. 2012, 41, 2718-39.

690 (8) Padamwar, M. N.; Pokharkar, V. B. Development of vitamin loaded 691 topical liposomal formulation using factorial design approach: Drug 692 deposition and stability. Int. J. Pharm. 2006, 320, 37-44.

693 (9) Taha, E. I. Lipid vesicular systems: formulation optimization and ex 694 vivo comparative study. J. Mol. Liq. 2014, 196, 211-216.

695 (10) Abdelbary, A. A.; AbouGhaly, M. H. H. Design and optimization 696 of topical methotrexate loaded niosomes for enhanced management of psoriasis: Application of Box-Behnken design, in-vitro evaluation and 697 in-vivo skin deposition study. Int. J. Pharm. 2015, 485, 235-243. 698

(11) Jadhav, S. M.; Morey, P.; Karpe, M.; Kadam, V. Novel vesicular 699 system: An overview. J. Appl. Pharm. Sci. 2012, 02, 193-202. 700

(12) Rajera, R.; Nagpal, K.; Singh, S. K.; Mishra, D. N. Niosomes: a 701 controlled and novel drug delivery system. Biol. Pharm. Bull. 2011, 34, 702 945-53.

(13) Uchegbu, I. F.; Vyas, S. P. Non-ionic surfactant based vesicles 704 (niosomes) in drug delivery. Int. J. Pharm. 1998, 172, 33-70. 705

(14) Bangham, A. D.; Standish, M. M.; Watkins, J. C. Diffusion of 706 univalent ions across the lamellae of swollen phospholipids. J. Mol. Biol. 707 1965, 13, 238-252.

(15) da Silva Malheiros, P.; Daroit, D. J.; Brandelli, A. Food 709 applications of liposome-encapsulated antimicrobial peptides. Trends 710 Food Sci. Technol. 2010, 21, 284-292.

(16) du Plessis, J.; Weiner, N.; Müller, D. G. The influence of in vivo 712 treatment of skin with liposomes on the topical absorption of a 713 hydrophilic and a hydrophobic drug in vitro. Int. J. Pharm. 1994, 103, 714 R1-R5.

(17) Manconi, M.; Sinico, C.; Valenti, D.; Loy, G.; Fadda, A. M. 716 Niosomes as carriers for tretinoin I: Preparation and properties. Int. J. 717 Pharm. 2002, 234, 237-248.

718

(18) Manca, M. L.; Manconi, M.; Nacher, A.; Carbone, C.; Valenti, D.; 719 Maccioni, A. M.; Sinico, C.; Fadda, A. M. Development of novel 720 diolein-niosomes for cutaneous delivery of tretinoin: Influence of 721 formulation and in vitro assessment. Int. J. Pharm. 2014, 477, 176-186. 722 (19) Mahale, N. B.; Thakkar, P. D.; Mali, R. G.; Walunj, D. R.; 723 Chaudhari, S. R. Niosomes: Novel sustained release nonionic stable 724 vesicular systems - An overview. Adv. Colloid Interface Sci. 2012, 183- 725 $184,46-54$.

(20) Moghassemi, S.; Hadjizadeh, A. Nano-niosomes as nanoscale 727 drug delivery systems: An illustrated review. J. Controlled Release 2014, 728 $185,22-36$.

(21) Mali, N.; Darandale, S.; Vavia, P. Niosomes as a vesicular carrier 730 for topical administration of minoxidil: formulation and in vitro 731 assessment. Drug Delivery Transl. Res. 2013, 3, 587-592.

(22) Fan, M.; Xu, S.; Xia, S.; Zhang, X. Preparation of salidroside nano- 733 liposomes by ethanol injection method and in vitro release study. Eur. 734 Food Res. Technol. 2008, 227, 167-174.

(23) Marianecci, C.; Di Marzio, L.; Rinaldi, F.; Celia, C.; Paolino, D.; 736 Alhaique, F.; Esposito, S.; Carafa, M. Niosomes from 80s to present: The 737 state of the art. Adv. Colloid Interface Sci. 2014, 205, 187-206. 738

(24) Akbarzadeh, A.; Rezaei-Sadabady, R.; Davaran, S.; Joo, S. W.; 739 Zarghami, N.; Hanifehpour, Y.; Samiei, M.; Kouhi, M.; Nejati-Koshki, K. 740 Liposome: classification, preparation, and applications. Nanoscale Res. 741 Lett. 2013, 8, 102.

(25) Justo, O. R.; Moraes, Â. M. Analysis of process parameters on the 743 characteristics of liposomes prepared by ethanol injection with a view to 744 process scale-up: Effect of temperature and batch volume. Chem. Eng. 745 Res. Des. 2011, 89, 785-792.

(26) Batzri, S.; Korn, E. D. Single bilayer liposomes prepared without 747 sonication. Biochim. Biophys. Acta, Biomembr. 1973, 298, 1015-1019. 748 (27) Pham, T. T.; Jaafar-Maalej, C.; Charcosset, C.; Fessi, H. Liposome 749 and niosome preparation using a membrane contactor for scale-up. 750 Colloid. Colloids Surf., B 2012, 94, 15-21.

(28) Loukas, Y. L. Experimental studies for screening the factors that 752 influence the effectiveness of new multicomponent and protective 753 liposomes. Anal. Chim. Acta 1998, 361, 241-251.

(29) Shah, S. R.; Parikh, R. H.; Chavda, J. R.; Sheth, N. R. Application 755 of Plackett-Burman screening design for preparing glibenclamide 756 nanoparticles for dissolution enhancement. Powder Technol. 2013, 235, 757 405-411.

(30) El-Samaligy, M. S.; Afifi, N. N.; Mahmoud, E. A. Increasing 759 bioavailability of silymarin using a buccal liposomal delivery system: 760 Preparation and experimental design investigation. Int. J. Pharm. 2006, 761 308, 140-148.

(31) Shaikh, K. S.; Chellampillai, B.; Pawar, A. P. Studies on nonionic 763 surfactant bilayer vesicles of ciclopirox olamine. Drug Dev. Ind. Pharm. 764 2010, 36, 946-53. 
766 (32) Mahmood, S.; Taher, M.; Mandal, U. K. Experimental design and 767 optimization of raloxifene hydrochloride loaded nanotransfersomes for 768 transdermal application. Int. J. Nanomed. 2014, 9, 4331-4346.

769 (33) Chaudhary, H.; Kohli, K.; Kumar, V. Nano-transfersomes as a 770 novel carrier for transdermal delivery. Int. J. Pharm. 2013, 454, 367-380.

771 (34) Derakhshandeh, K.; Erfan, M.; Dadashzadeh, S. Encapsulation of 772 9-nitrocamptothecin, a novel anticancer drug, in biodegradable 773 nanoparticles: Factorial design, characterization and release kinetics. 774 Eur. J. Pharm. Biopharm. 2007, 66, 34-41.

775 (35) Alund, S. J.; Smistad, G.; Hiorth, M. A. A multivariate analysis 776 investigating different factors important for the interaction between 777 liposomes and pectin. Colloids Surf., A 2013, 420, 1-9.

778 (36) Gonzalez-Mira, E.; Egea, M. A.; Garcia, M. L.; Souto, E. B. Design 779 and ocular tolerance of flurbiprofen loaded ultrasound-engineered NLC. 780 Colloids Surf., B 2010, 81, 412-421.

781 (37) Pando, D.; Caddeo, C.; Manconi, M.; Fadda, A. M.; Pazos, C. 782 Nanodesign of olein vesicles for the topical delivery of the antioxidant 783 resveratrol. J. Pharm. Pharmacol. 2013, 65, 1158-1167.

784 (38) Pando, D.; Matos, M.; Gutiérrez, G.; Pazos, C. Formulation of 785 resveratrol entrapped niosomes for topical use. Colloids Surf., B 2015, $786128,398-404$

787 (39) Devaraj, G. N.; Parakh, S. R.; Devraj, R.; Apte, S. S.; Rao, B. R.; 788 Rambhau, D. Release Studies on Niosomes Containing Fatty Alcohols 789 as Bilayer Stabilizers Instead of Cholesterol. J. Colloid Interface Sci. 2002, $790251,360-365$.

791 (40) Kremer, J. M. H.; Van der Esker, M. W.; Pathmamanoharan, C.; 792 Wiersema, P. H. Vesicles of variable diameter prepared by a modified 793 injection method. Biochemistry 1977, 16, 3932-3935.

794 (41) Pons, M.; Foradada, M.; Estelrich, J. Liposomes obtained by the 795 ethanol injection method. Int. J. Pharm. 1993, 95, 51-56.

796 (42) Justo, O. R; Moraes, A. M. Kanamycin incorporation in lipid 797 vesicles prepared by ethanol injection designed for tuberculosis 798 treatment. J. Pharm. Pharmacol. 2005, 57, 23-30.

799 (43) Szoka, F. C., Jr. Preparation of liposome and lipid complex 800 compositions. U.S. Patent 5,549,910, 1996.

801 (44) Ghanbarzadeh, S.; Arami, S. Enhanced Transdermal Delivery of 802 Diclofenac sodium via conventional liposomes, ethosomes, and 803 transfersomes. BioMed Res. Int. 2013, 2013, 1-7.

804 (45) Antonietti, M.; Förster, S. Vesicles and liposomes: a self-assembly 805 principle beyond lipids. Adv. Mater. 2003, 15, 1323-1333.

806 (46) Wang, Z.; He, X. Dynamics of vesicle formation from lipid 807 droplets: Mechanism and controllability. J. Chem. Phys. 2009, 130, 808094905 .

809 (47) Lasic, D. D. The mechanism of vesicle formation. Biochem. J. $8101988,256,1-11$.

811 (48) Janmey, P. A.; Kinnunen, P. K. J. Biophysical properties of lipids 812 and dynamic membranes. Trends Cell Biol. 2006, 16, 538-546.

813 (49) Silva, R.; Ferreira, H.; Little, C.; Cavaco-Paulo, A. Effect of 814 ultrasound parameters for unilamellar liposome preparation. Ultrason. 815 Sonochem. 2010, 17, 628-632.

816 (50) Yamaguchi, T.; Nomura, M.; Matsuoka, T.; Koda, S. Effects of 817 frequency and power of ultrasound on the size reduction of liposome. 818 Chem. Phys. Lipids 2009, 160, 58-62.

819 (51) Lo, C. T.; Jahn, A.; Locascio, L. E.; Vreeland, W. N. Controlled 820 self-assembly of monodisperse niosomes by microfluidic hydrodynamic 821 focusing. Langmuir 2010, 26, 8559-8566. 